\title{
Serum levels of the interferon- $\gamma$-inducing cytokine interleukin-18 are increased in individuals at high risk of developing Type I diabetes
}

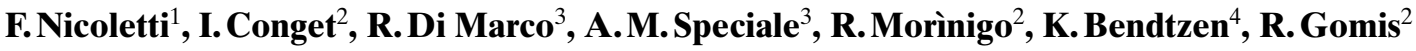 \\ ${ }^{1}$ Department of Clinical Medicine, Prevention and Biotechnological Health, University of Milan Bicocca, Italy \\ ${ }^{2}$ Endocrinology and Diabetes Unit, Hospital Clinic, Faculty of Medicine, University of Barcelona, IDIBAPS \\ (Institute of Biomedical Studies August Pri i Sunyer) Barcelona, Spain \\ ${ }^{3}$ Department of Microbiological and Gynecological Sciences, University of Catania, Italy \\ ${ }^{4}$ Institute for Inflammation Research, Rigshospitalet University Hospital, Copenhagen, Denmark
}

\section{Abstract}

Aims/hypothesis. Interleukin (IL)-18 is a cytokine primarily produced by macrophages and capable of inducing $\mathrm{T}$ lymphocyte synthesis of interferon (IFN) $-\gamma$. An up-regulated synthesis of IFN- $\gamma$ with consequential Type I cytokine dominance has been repeatedly shown in Type I (insulin-dependent) diabetes mellitus and thought to be involved in its pathogenesis. Because increased production of IFN- $\gamma$ could be secondary to a dysregulated synthesis of IL18 , we compared the circulating levels of IL-18 in patients with newly diagnosed Type I diabetes with those of non-diabetic first-degree relatives and healthy control subjects.

Methods. Serum samples were obtained from healthy control subjects, patients with newly diagnosed Type I diabetes, and their healthy first-degree relatives. The latter were subdivided into "low" and "high" risk prediabetics depending on whether they were negative or positive for two or more of the anti-pancreatic autoantibodies ICA, GAD, IA-2 and IAA. Serum levels of IL-18 were measured by solid-phase ELISA.

Results. Interleukin (IL)-18 was above the detection limit of $25 \mathrm{pg} / \mathrm{ml}$ in 7 of $40(17 \%)$ healthy control subjects, in 5 of $35(14 \%)$ patients and in 3 of $30(10 \%)$ first-degree relatives at low risk of developing Type I diabetes. In contrast, IL-18 could be detected in 19 of $28(68 \% ; p<0.0001)$ relatives at high risk. The mean serum level of IL-18 was higher in these individuals when compared with the low-risk relatives, patients and control subjects.

Conclusion/interpretation. IL-18 serum levels are increased selectively during the early, subclinical stage of Type I diabetes. [Diabetologia (2001) 44: 309-311]

Keywords Interleukin-18, serum levels, interferon- $\gamma$
Type 1 proinflammatory cytokines such as interferon(IFN)- $\gamma$, interleukin (IL)-1 $\beta$, IL-12 and tumour necrosis factor (TNF)- $\alpha$ released by macrophage and $\mathrm{T}$ lymphocytes in the vicinity of pancreatic beta cells have repeatedly been implicated in the pathogenesis of Type I (insulin-dependent) diabetes mellitus [1].

Received: 25 July 2000 and in revised form: 12 October 2000

Corresponding author: F. Nicoletti, MD, Via Luigi Sturzo n.3, 95021, Cannizzaro, Catania, Italy

Abbreviations: aAb, autoantibodies; GAD Ab, glutamic acid decarboxylase antibodies ; IAA, insulin autoantibodies; IA2 $\mathrm{Ab}$, tyrosine phosphatase antibodies ICA, islet cell antibodies; IFN, interferon.
IL-18 is another type 1 cytokine primarily produced by macrophages and closely related to the IL1 family of cytokines [1]. It has been shown to play a pivotal part in the generation of type 1 cytokine responses through its ability with IL-12 to up-regulate IFN- $\gamma$ production from T cells and natural killer cells. An up-regulated production of IL-18 could therefore be an important pathogenic event in the dysregulated production of IFN- $\gamma$ and other type 1 cytokines thought to predispose to immunoinflammatory diseases such as Type I diabetes.

These observations prompted us to study the circulating levels of IL-18 in a group of patients with newly diagnosed Type I diabetes along with their healthy first-degree relatives. The latter were arbitrarily classified as at "low" and "high" risk for dis- 
ease development depending on whether they were negative or positive for two or more anti-pancreatic autoantibodies $(\mathrm{aAb})$, including islet cell antibodies (ICA), anti-glutamic acid decarboxylase antibodies (GAD Ab), tyrosine phosphatase antibodies (IA2 $\mathrm{Ab}$ ) and insulin $\mathrm{aAb}$ (IAA).

\section{Materials and methods}

Subjects and blood sampling. The study groups consisted of one group with 35 ( 20 women) patients with Type I diabetes recruited within 6 weeks of diagnosis. The mean age was 23.3 years (range: 18 to 37 years). A second group with 28 (18 women) high risk relatives of Type I diabetic patients, still normoglycaemic and without clinical or laboratory criteria of the disease. The mean age was 24.5 years (range: 18 to 44 years). A third group with 30 (20 women) low risk relatives of Type I diabetic patients. The mean age was 24.7 years (range: 16 to 43 years). A group of 40 subjects ( 22 women) was matched with healthy control subjects for sex and age. The mean age was 24.5 years (range: 16 to 43 years).

None of the subjects suffered from infectious, allergic or other autoimmune diseases in the 6 months before sampling, and they did not use immunomodulatory drugs in the 3 months preceding the study.

Venous blood from fasting subjects were collected between 9.00 and $10.00 \mathrm{a} . \mathrm{m}$. to minimize possible circadian variations. Blood samples were allowed to clot at room temperature and the sera were immediately separated by centrifugation at 1000 $\mathrm{x} \mathrm{g}$ and stored at $-20^{\circ} \mathrm{C}$ until assayed.

IL-18 assay. We measured IL-18 by specific ELISA using a mouse anti-human IL-18 monoclonal antibody for capturing the cytokine and a biotinylated polyclonal rabbit anti-human IL-18 antibody for detection (both from R \& D Systems, Minneapolis, Minn., USA). Recombinant human IL-18 (PeproTech, London, UK) was used as standard. The ELISA was carried out as described elsewhere for other cytokines [2]. The sensitivity limit of the assay was $25 \mathrm{pg} / \mathrm{ml}$. The intra-assay and inter-assay coefficients of variations were $6.5 \%$ and $9 \%$, respectively.

To rule out the possibility that heterophilic antibodies interfered with the ELISA kits, control experiments were carried out in which $100 \mu \mathrm{g}$ of irrelevant mouse IgG (Sigma Chimica, Milan, Italy) were added to the sera in a final volume of $10 \mu \mathrm{l}$ phosphate-buffered saline (PBS) as described elsewhere [2]. Control samples were treated with $10 \mu \mathrm{l}$ PBS alone.

Anti-pancreatic beta-cell antibody assays. Glutamic acid decarboxylase antibodies (GADAb) were determined by a radiobinding assay [3] and were considered positive when above 2 $\mathrm{U} / \mathrm{ml}$. The assay for GADAb achieved in the second GAD proficiency test a $100 \%$ sensitivity and $100 \%$ specificity. Tyrosine phosphatase antibodies (IA2Ab) titres were measured by a radioimmunoassay as described previously [4] and considered positive when above $0.8 \mathrm{U} / \mathrm{ml}$. The upper limits of normal values for GADAb and IA2Ab were defined by the 99th centile of antibodies measured in 110 non-diabetic subjects without familial history of Type I diabetes. Insulin autoantibodies (IAA) were measured using a radiobinding method [5]. The inter-assay and intra-assay coefficients of variation for IA 2 determination were $7 \%$ and $5 \%$ respectively. The upper normal limit, $1 \%$, was defined after the analysis of 500 samples from healthy control subjects. Inter-assay coefficients of variation

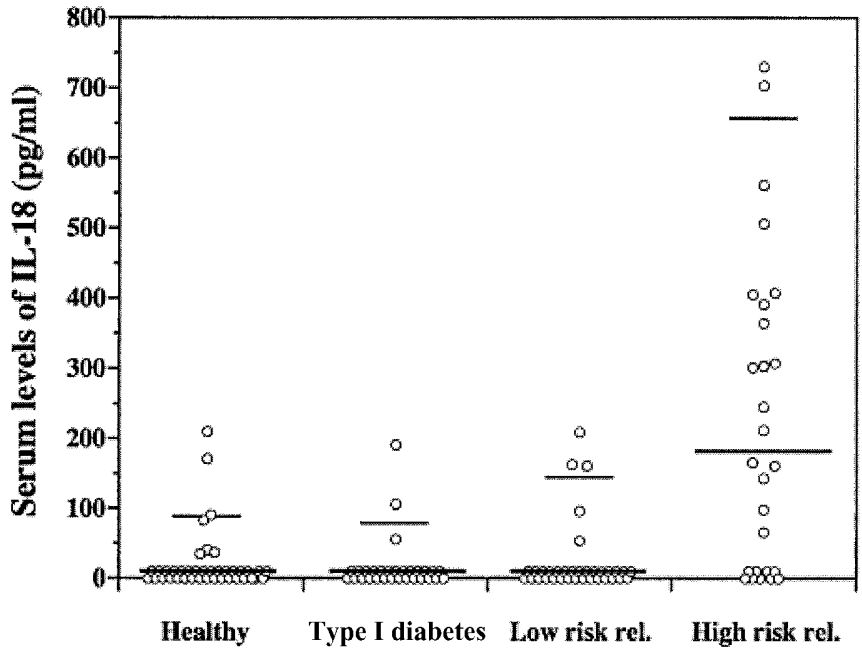

Fig. 1. Increased IL-18 serum levels in subjects with Type I prediabetes. Serum levels were measured by solid-phase ELISA in healthy control subjects, in patients with newly diagnosed Type I diabetes, in their healthy first-degree relatives which were either negative (low-risk relatives) or positive (high-risk relatives) for two or more anti-pancreatic antibodies (see Methods section). Horizontal lines show the medians and $95 \%$ centiles, respectively). The sensitivity limit of the IL-18 assay was $25 \mathrm{pg} / \mathrm{ml}$. The number of high-risk relatives with levels of IL-18 over $25 \mathrm{pg} / \mathrm{ml}$ differed significantly from those in each of the other groups ( $p<0.0001$, Fisher's exact test). The serum levels of IL-18 in these groups also differed significantly from those in the other groups $(p<0.0001$, Mann-Whitney rank sum test)

for IAA were $12 \%$. ICA levels were measured by a standard two-step indirect immunofluorescence assay on unfixed cryostat sections of blood group 0 human pancreases [6]. Samples with more than 5 Juvenile Diabetes Foundation units were considered positive.

\section{Results}

As shown in Figure 1, IL-18 was found in the serum of only a minority of the healthy control subjects, the patients with newly diagnosed Type I diabetes, and the first-degree relatives of Type I diabetes patients at low risk of developing Type I diabetes. In contrast, IL-18 was detected in $68 \%$ of the relatives at high risk of developing the disease. These subjects also had higher serum levels of IL-18 than the other groups. Addition of mouse IgG failed to modify the levels of IL-18 in subjects with detectable levels of IL-18 and did not change the optical densities of the negative samples (data not shown). The differences in read-out between mouse IgG-treated samples and untreated samples were within the range of the intra-assay and inter-assay coefficients of variation (6.5\% and $9 \%$, respectively). These control experiments were carried out to rule out the possibility that the higher serum levels of IL-18 in high-risk individuals were caused by heterophile Abs, as has been 
suggested by cases where high levels of IL-4 in Type I diabetes appear to have been found [7]. The number of IL-18 positives and the levels of the cytokine did not correlate with sex or age, or with the presence or titres of anti-pancreatic aAbs.

\section{Discussion}

These results show for the first time that circulating levels of IL-18 increased in relatives of Type I diabetes patients with anti-pancreatic beta-cell autoimmunity as defined by the presence of two or more of the autoantibodies ICA, GAD Ab, IA2 Ab and IAA. The clinical validity of this seroimmunological subdivision was substantiated by the observation that during a 5 year follow-up period conducted in 16 of the 28 high-risk relatives and in 15 of the 30 low-risk relatives, Type I diabetes occurred in 12 of the 16 highrisk relatives and in none of the 15 low-risk relatives. Interestingly, IL-18 was detectable in 14 of the 16 subjects when sampled during the prediabetic period, but the cytokine could be measured only in 4 of the 16 subjects at the time of the clinical onset of Type I diabetes ( $p=0.001$, Fisher's exact test). Thus, IL-18 appears to increase during the prediabetes period but not at the onset of the disease.

Generally accepted concepts of the development of Type I diabetes indicate that the pathogenic contribution of IL-18 occurs through its ability to stimulate T-cell synthesis of IFN- $\gamma$. Increased production of IFN- $\gamma$ has indeed been observed in peripheral blood mononuclear cells from high-risk first-degree relatives of patients with Type I diabetes [8]. Subsequently, when the production of IFN- $\gamma$ is sufficiently upregulated, the cytokine could itself become capable of sustaining its own increased production by stimulating IFN- $\gamma$ producing T cells. This could in turn potentiate and prolong the type 1 cytokine profile observed at the onset of Type I diabetes which is characterized by increased circulating levels of IFN- $\gamma$, IL-2 and TNF- $\alpha$ [6]. In agreement with this scenario of Th1 dominance and defective Th2 activity in the prediabetic phase, other investigators have reported a higher production of Th2 antiinflammatory cytokines from the blood mononuclear cells of twins at a lowrisk of disease development when compared with their diabetic twins [9]. It is possible that the differences between the findings before and after diagnosis could be related to non-immunological causes such as hyperglycaemia or other metabolic derangements in newly diagnosed diabetes patients. This, however, does not seem likely because preliminary data show that IL-18 is detectable in only 3 of 20 patients with Type II (non-insulin-dependent) diabetes mellitus who undergo insulin therapy, a percentage very similar to that observed in healthy control subjects and in low-risk Type I diabetic relatives.
That IL-18 could play a part in the pathogenesis of Type I diabetes is also supported by in vivo studies in the NOD mouse model of Type I diabetes showing that intrainsular expression of IL-18 mRNA coincides with, and perhaps promotes, the onset of destructive insulitis [1]. This and the present results and the known proinflammatory activities of IL-18 in vitro and in vivo [1] are difficult to reconcile with the capacity of exogenous IL-18 to prevent disease development in NOD mice [10]. Results from a systemic administration of cytokines in NOD mice might not, however, have direct pathogenic implications. Compensatory mechanisms, such as the induction of cytokine antagonists, could be triggered by a systemic administration of cytokines. Furthermore, injecting NOD mice with large amounts of IL18 might not mirror the pathophysiological action of minute amounts of IL-18 released at the beta-cell level. In fact, continuous treatment of NOD mice with IL-18 has been shown to inhibit diabetes development by preventing the sex and age-related progression of a Th2-dependent benign insulitis to an aggressive Th1-dependent form [10].

\section{References}

1. Dinarello CA (1999) IL-18: A Th1-inducing, proinflammatory cytokine and new member of the IL-1 family. J Allergy Clin. Immunol, 103: 11-24

2. Nicoletti F, Patti F, Cocuzza C et al. (1996) Elevated serum levels of interleukin-12 in chronic progressive multiple sclerosis. J Neuroimmunol 70: 87-90

3. Luhder F, Woltanski KP, Mauch L et al. (1994) Detection of autoantibodies to the $65-\mathrm{kD}$ isoform of glutamate decarboxylase by radioimmunoassay. Eur J Endocrinol 130: 575-580

4. Masuka M, Powell M, Chen Set al. (2000) Autoantibodies to IA-2 in insulin-dependent diabetes mellitus. Clin Chim Acta 291: 53-66

5. Vardi P, Dib SA, Tuttleman M et al. (1987) Competitive insulin autoantibody assay. Prospective evaluation of subjects at high-risk for development of Type I diabetes mellitus. Diabetes 36: 1286-1291

6. Hussain MJ, Peakman M, Gallati H et al. (1996) Elevated serum levels of macrophage-derived cytokines precede and accompany the onset of IDDM. Diabetologia, 39: 60-69

7. Redondo MJ, Gottlieb PA, Motheral T et al. (1999) Heterophile anti-mouse immunoglobulin antibodies could interfere with cytokine measurements in patients with HLA alleles protective for Type IA diabetes. Diabetes 48: 2166-2170

8. Karlsson MGE, Sederholm Lawesson S, Ludvigsson J (2000) Th-1-like dominance in high-risk first-degree relatives of Type I diabetic patients. Diabetologia 43: 742-749

9. Kalmann BA, Lampeter EF, Hanfi-Moghaddam P, Hawa M, Leslie RD, Kolb H (1999) Cytokine secretion patterns in twins discordant for Type I diabetes. Diabetologia 42: 1080-1085

10. Rothe H, Hausmann A, Casteels K et al. (1999) IL-18 inhibits diabetes development in nonobese diabetic mice by counterregulation of Th1-dependent destructive insulitis. J Immunol 163: 1230-1236 